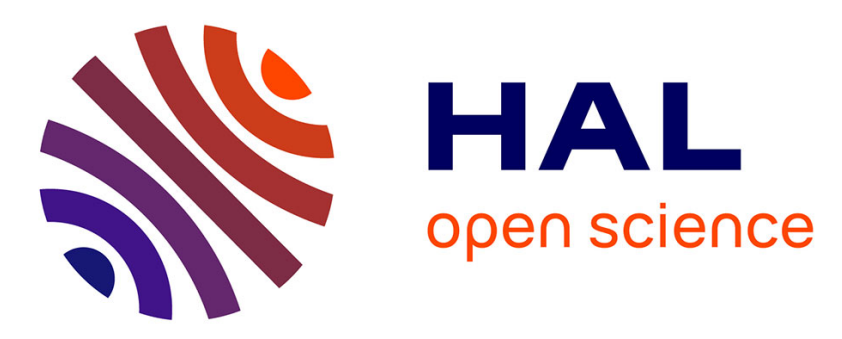

\title{
Indoor localization using polar intervals in wireless sensor networks
}

Farah Mourad-Chehade, Paul Honeine, Hichem Snoussi

\section{To cite this version:}

Farah Mourad-Chehade, Paul Honeine, Hichem Snoussi. Indoor localization using polar intervals in wireless sensor networks. Proc. 19th International Conference on Telecommunications (ICT), 2012, Jounieh, Lebanon. pp.1 - 6, 10.1109/ICTEL.2012.6221222 . hal-01966018

\section{HAL Id: hal-01966018 https://hal.science/hal-01966018}

Submitted on 27 Dec 2018

HAL is a multi-disciplinary open access archive for the deposit and dissemination of scientific research documents, whether they are published or not. The documents may come from teaching and research institutions in France or abroad, or from public or private research centers.
L'archive ouverte pluridisciplinaire HAL, est destinée au dépôt et à la diffusion de documents scientifiques de niveau recherche, publiés ou non, émanant des établissements d'enseignement et de recherche français ou étrangers, des laboratoires publics ou privés. 


\title{
Indoor localization using polar intervals in wireless sensor networks
}

\author{
Farah Mourad, Paul Honeine, Hichem Snoussi \\ ICD-LM2S (UMR STMR CNRS 6279) \\ Université de Technologie de Troyes \\ 12, rue Marie Curie, 10010, France \\ Email: \{farah.mourad, paul.honeine, hichem.snoussi\}@utt.fr
}

\begin{abstract}
Wireless sensor networks are networks composed of a large number of distributed sensors, connected via wireless links. This paper deals with the problem of localization in wireless sensor networks. Such a problem becomes challenging in indoor environments, where signals of Global Positioning Systems are no more reliable. In this paper, the localization problem is defined using connectivity measurements. The proposed technique consists thus of estimating unknown sensors positions using known position information of neighboring sensors. The estimation is then performed using polar intervals. The estimated positions are thus two-dimensional intervals defined in some polar coordinates system. Using intervals, the proposed approach performs an outer estimation of the solution, leading to estimates covering for sure the actual positions of the sensors.
\end{abstract}

Index Terms - connectivity measurements, localization problem, polar intervals, wireless sensor network.

\section{INTRODUCTION}

In recent years, advances in electronics and wireless communication have produced a new technological innovation, the Wireless Sensor Networks (WSNs). These networks are composed of a large number of embedded devices having sensing, computing and communication capabilities [1], [2]. Due to their wireless links, sensors in WSNs do not have a fixed infrastructure and thus they are able to move [3], [4]. Their mobility could be either passive or controlled. In the case of passive mobility, sensors change positions in an uncontrollable manner, and thus they need to be localized regularly, whereas in the case of controlled mobility, sensors are robots whose mobility could be managed to improve the accuracy of the sensed data in the network [5], [6].

WSNs have emerged as a feasible solution for a wide range of applications in military, environment monitoring, healthcare, etc [7], [8]. In almost all applications, the knowledge of sensors locations is essential, since sensed data are directly related to the place where measurements are made. This paper considers the problem of sensors localization in uncontrolled mobility sensor networks. The self-evident solution to such a problem consists of using Global Positioning Systems (GPS) [9]. However, this solution is impractical for indoor localization since in indoor environments, the reception of GPS signals is no more reliable. The alternative solution consists of considering two types of sensors, anchors and non-anchor nodes. Anchors are sensors aware of their locations, whereas non-anchor nodes, or simply nodes, have unknown locations and thus they need to be localized regularly. Anchors are generally static, having pre-defined fixed positions, or mobile but tracked or moved by the user.

Many algorithms have been proposed for indoor localization using anchors. They are mainly based on estimating the distances separating the nodes from the anchors, using Time Of Arrival (TOA) [10], Time Difference Of Arrival (TDOA) [11], Angle Of Arrival (AOA) [12], or Received Signal Strength Indicator (RSSI) [13]. TOA-based methods measure the travel times of signals exchanged between the sensors, whereas TDOA and AOA methods measure the difference of arrival times or the angles at reception of exchanged signals. While an error in synchronization can significantly falsify the estimation using TOA methods, TDOA and AOA methods achieve high estimation accuracy but need extra hardware. Compared to other methods, the RSSI methods are simpler and inexpensive. They are based on the attenuation of signal strengths with the increase of the traveled distance.

Localization using RSSI-based methods is challenging because of reflection, diffraction and scattering of signals. However, it has drawn considerable attention due to its lowcomplexity in calculation and a very low cost of implementation. For instance, in [14], [15], Monte-Carlo-based techniques are proposed for sensors localization using RSSI information. In [16], authors propose a statistical method for distance estimation also based on RSSI. A Sigma-Point Kalman Smoothers-based method is proposed for RSSI-based localization in [17]. In [18], [19], the variational filter and the quantized variational filter are used for sensors radiolocalization. In a different scenario, interval-based methods using RSSI measurements are proposed in [20], [21], [6]. Compared to other approaches, these techniques perform an outer approximation of the solution area, leading to boxes guaranteed to include the actual locations.

This contribution proposes an original approach for indoor localization in wireless sensor networks. The proposed approach is an RSSI-anchor-based method, using connectivity measurements. In other words, measurements are proximity information setting whether the considered node is within the sensing range of other anchors or not. The localization problem is solved using polar intervals. Based on interval analysis [22], [21], the method aims at computing bounds on sensors coordinates, in the Polar Coordinate System (PCS). 
The estimated positions are partial rings including for sure all the possible solutions of the problem. Simulation results using Matlab corroborate the efficiency of the proposed method compared to other Monte-Carlo-based and interval-based techniques.

The rest of the paper is organized as follows. Section II introduces the localization problem. Section III describes the proposed algorithm to solve the problem. Simulation results are given in Section IV whereas Section V concludes the paper.

\section{Problem statement}

Consider a network composed of $N_{a}$ anchors and $N_{u}$ nodes and let $\boldsymbol{a}_{i}(t), i \in\left\{1, \ldots, N_{a}\right\}$, and $\boldsymbol{u}_{j}(t), j \in\left\{1, \ldots, N_{u}\right\}$, be the positions of these sensors respectively. The aim of the method is to estimate all $\boldsymbol{u}_{j}(t)$ positions using $\boldsymbol{a}_{i}(t)$ information. In order to reduce the communication costs, the proposed method assumes that nodes exchange information only with anchors. For this reason and without loss of generality, only one node $\boldsymbol{u}(t)$ is considered in this paper and the index $j$ is withdrawn. Assume that all sensors are deployed in a twodimensional square area, then their coordinates in the PCS are given by $\boldsymbol{a}_{i}(t)=\left(\rho_{i}(t), \theta_{i}(t)\right)$ and $\boldsymbol{u}(t)=(\rho(t), \theta(t))$, where $\rho$ denotes the distance from the origin to the sensor and $\theta$ denotes the angle measured anticlockwise from the $\mathrm{x}$-axis to the line joining the sensor to the origin. The origin of the PCS, called also pole, is assumed to be at the low-left corner of the surveillance area. Then $\theta_{i}(t) \in\left[0, \frac{\pi}{2}\right], \theta(t) \in\left[0, \frac{\pi}{2}\right]$, $\rho_{i}(t) \in\left[0, \rho_{\max }\right]$ and $\rho(t) \in\left[0, \rho_{\max }\right]$, where $\rho_{\max }$ is the length of the diagonal of the surveillance area.

The proposed method uses RSSI information to localize the mobile node. Indeed, at each time step, every anchor broadcasts signals in the network with the same initial power. According to the Okumura-Hata model [23], [24], the strengths of the signals decrease monotonically with the increase of their traveled distances as follows,

$$
\xi_{i}(t)=\xi_{0}\left(\frac{d_{0}}{d_{i}(t)}\right)^{\alpha},
$$

where $\xi_{i}(t)$ is the strength of the signal emitted by the anchor $i$ and received by the mobile node at time $t, \xi_{0}$ is the strength measured at a reference distance $d_{0}$ from the anchor $i, d_{i}(t)=$ $\left\|\boldsymbol{u}(t)-\boldsymbol{a}_{i}(t)\right\|$ is the Euclidian distance between the anchor $i$ and the node at time $t$ and $\alpha$ is the path loss exponent.

In practice, the RSSI of a signal could be modified due to the reflection, the diffraction or the scattering of the signal. Moreover the values of $\xi_{0}$ and $\alpha$ may vary from an anchor to the other. This may lead to inaccurate distances estimates. For this reason, the proposed method uses connectivity information, instead of using distances estimates. Here, received strength values are only used to be compared to a threshold $\xi_{r}$, corresponding to the sensing range $r$ of the sensors. If $\xi_{i}(t) \geq \xi_{r}$, the anchor $i$ is assumed to be within the sensing range of the node at time $t$. Otherwise, the anchor $i$ is assumed to be too far and its information is not used. Connectivity mea-

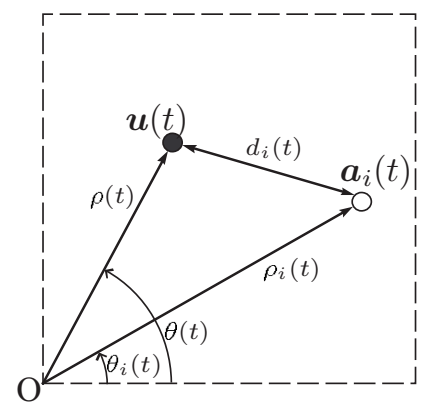

Fig. 1. Coordinates in the PCS.

surements are then one-bit information generated as follows,

$$
y_{i}(t)=\left\{\begin{array}{ll}
1 & \text { if } \xi_{i}(t) \geq \xi_{r} \\
0 & \text { otherwise }
\end{array} \quad, \quad i \in\left\{1, \ldots, N_{a}\right\} .\right.
$$

Let $I(t)$ be the set of indices at time $t$ of all anchors having $y_{i}(t)=1$. The anchors denoted in $I(t)$ are assumed to be within the sensing range of the node, and thus they are located at distances from the node less than $r$,

$$
\forall i \in I(t), d_{i}(t) \leq r .
$$

One could obtain the distance $d_{i}(t)$ between the anchor $i$ and the node $\boldsymbol{u}$ by considering the triangle having the origin $\mathrm{O}, \boldsymbol{a}_{i}(t)$ and $\boldsymbol{u}(t)$ as vertices, as shown in Fig. 1. The lengths of the sides $\mathrm{O} \boldsymbol{u}(t)$ and $\mathrm{Oa}_{i}(t)$ are given by $\rho(t)$ and $\rho_{i}(t)$ respectively, whereas $d_{i}(t)$ is the length of the side $\boldsymbol{u}(t) \boldsymbol{a}_{i}(t)$ in the triangle. $d_{i}(t)$ could then be computed using the generalized Pythagorean theorem as follows,

$$
d_{i}^{2}(t)=\rho^{2}(t)+\rho_{i}^{2}(t)-2 \rho(t) \rho_{i}(t) \cos \left(\theta(t)-\theta_{i}(t)\right),
$$

with $\left|\theta(t)-\theta_{i}(t)\right|$ being the angle at the vertex O.

The localization problem is then defined in the PCS by the following constraints,

$$
\rho^{2}(t)+\rho_{i}^{2}(t)-2 \rho(t) \rho_{i}(t) \cos \left(\theta(t)-\theta_{i}(t)\right) \leq r^{2}, i \in I(t) .
$$

Graphically, the problem at a given time $t$ consists of a set of disks having $r$ as radii and the anchors $i, i \in I(t)$, as centers. An example of such a problem with three detected anchors is shown in Fig. 2. The solution of the problem is given by the overlapping area of all disks, as shown in dark gray in the plot.

\section{POLAR-INTERVAL LOCALIZATION ALGORITHM}

Solving the localization problem consists of estimating the coordinates of the considered node $\boldsymbol{u}(t)=(\rho(t), \theta(t))$ at each time step, given the following constraints,

$\rho^{2}(t)+\rho_{i}^{2}(t)-2 \rho(t) \rho_{i}(t) \cos \left(\theta(t)-\theta_{i}(t)\right) \leq r^{2}, i \in I(t)$,

where $\rho_{i}(t), \theta_{i}(t)$, for $i \in I(t)$, and $r$ are known quantities. 


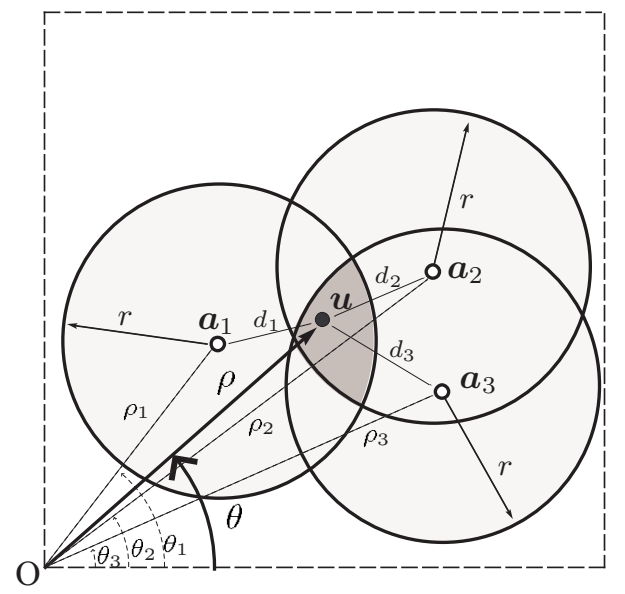

Fig. 2. An example of a localization problem at a given time step.

\section{A. Description of the method}

The solution of the problem is proposed using interval analysis [22]. Instead of computing a punctual position estimate at each time step, the method consists of performing an outer estimation of the solution. In other words, it aims at bounding the coordinates of the node, in the way to cover all possible solutions of the problem. The solution is then given by a twodimensional interval, denoted by $[\boldsymbol{u}](t)$. Also called polar box, $[\boldsymbol{u}](t)$ is defined by the cartesian product of two real intervals $[\rho](t)$ and $[\theta](t)$, defined over the polar coordinates $\rho(t)$ and $\theta(t)$ respectively,

$$
[\boldsymbol{u}](t)=[\rho](t) \times[\theta](t)=[\underline{\rho}(t), \bar{\rho}(t)] \times[\underline{\theta}(t), \bar{\theta}(t)],
$$

where $\rho(t)=\inf ([\rho](t))$ and $\bar{\rho}(t)=\sup ([\rho](t))$ denote respectively the lower and the higher endpoints of the real interval $[\rho](t)$ and $\underline{\theta}(t)=\inf ([\theta](t))$ and $\bar{\theta}(t)=\sup ([\theta](t))$ denote respectively the lower and the higher endpoints of the real interval $[\theta](t)$. Having the localization problem of (6) at time $t$, solving the problem consists of finding the minimal polar box $[\boldsymbol{u}](t)$ including all possible solutions. Starting with an initial box $[\boldsymbol{u}]_{0}$, the proposed method aims at minimizing the widths $(\bar{\rho}(t)-\underline{\rho}(t))$ and $(\bar{\theta}(t)-\underline{\theta}(t))$ of $[\rho](t)$ and $[\theta](t)$ respectively according to the constraints of (6). The initial polar box $[\boldsymbol{u}]_{0}$ could be defined by $\left[0, \rho_{\max }\right] \times\left[0, \frac{\pi}{2}\right]$, since $\rho(t) \in\left[0, \rho_{\max }\right]$ and $\theta(t) \in\left[0, \frac{\pi}{2}\right]$ as shown in Section II. Graphically, the solution box is a partial ring, having the origin of the PCS as center and $\underline{\rho}(t)$ and $\bar{\rho}(t)$ as inner and outer radii respectively, and defined between the lines starting at the origin and having $\underline{\theta}(t)$ and $\bar{\theta}(t)$ as angles from the $\mathrm{x}$-axis. The best solution box corresponding to the problem of Fig. 2 is given in thick black line in Fig. 3.

\section{B. Proposed algorithm}

In order to compute the solution box $[\boldsymbol{u}](t)$ at a given time $t$, one should set all available constraints on $\rho(t)$ and $\theta(t)$ according to (6). The first general constraints to be set are

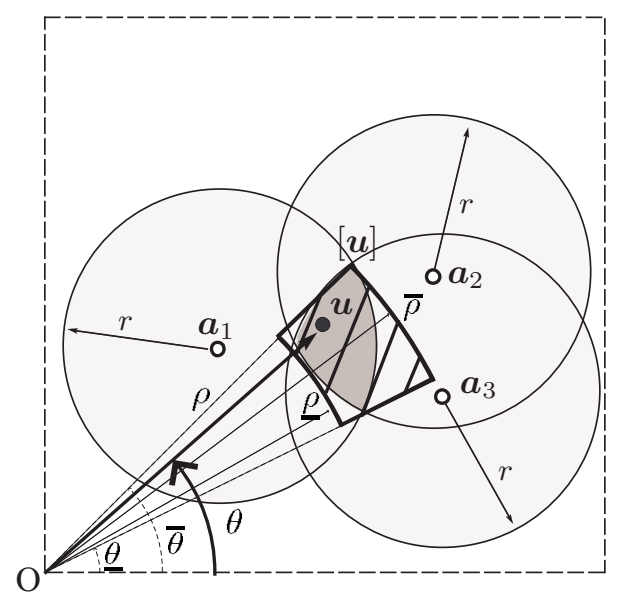

Fig. 3. Polar box obtained for the localization problem of Fig. 2.

given by the dimensions of the surveillance area,

$$
0 \leq \rho(t) \leq \rho_{\max } \quad \text { and } \quad 0 \leq \theta(t) \leq \frac{\pi}{2} .
$$

Moreover, since the cosine of an angle is always less than 1 and $\rho(t)$ and $\rho_{i}(t)$ are always positive for $i \in I(t)$, then $\left(\rho(t)-\rho_{i}(t)\right)^{2} \leq \rho^{2}(t)+\rho_{i}^{2}(t)-2 \rho(t) \rho_{i}(t) \cos \left(\theta(t)-\theta_{i}(t)\right)$. Each constraint of (6), for $i \in I(t)$, leads hence to the following constraint,

$$
\left(\rho(t)-\rho_{i}(t)\right)^{2} \leq r^{2} \Leftrightarrow \rho_{i}(t)-r \leq \rho(t) \leq \rho_{i}(t)+r .
$$

Then, if all constraints over $\rho(t)$ are combined, one obtains

$$
\max \left(0, \max _{i \in I(t)}\left(\rho_{i}(t)-r\right)\right) \leq \rho(t) \leq \min \left(\rho_{\max }, \min _{i \in I(t)}\left(\rho_{i}(t)+r\right)\right) .
$$

On the other hand,

$$
\begin{gathered}
\rho^{2}(t)+\rho_{i}^{2}(t)-2 \rho(t) \rho_{i}(t) \cos \left(\theta(t)-\theta_{i}(t)\right)= \\
\left(\rho(t)-\rho_{i}(t) \cos \left(\theta(t)-\theta_{i}(t)\right)\right)^{2}+\rho_{i}^{2}(t) \sin ^{2}\left(\theta(t)-\theta_{i}(t)\right) .
\end{gathered}
$$

Then each constraint of (6) leads inevitably to the following,

$$
\rho_{i}^{2}(t) \sin ^{2}\left(\theta(t)-\theta_{i}(t)\right) \leq r^{2} \Leftrightarrow-\frac{r}{\rho_{i}(t)} \leq \sin \left(\theta(t)-\theta_{i}(t)\right) \leq \frac{r}{\rho_{i}(t)} .
$$

If $\frac{r}{\rho_{i}(t)} \leq 1$, for $i \in I(t)$, and since $\left(\theta(t)-\theta_{i}(t)\right) \in\left[-\frac{\pi}{2}, \frac{\pi}{2}\right]$ where the sine function is monotonically increasing, the previous constraint leads to

$$
\theta_{i}(t)-\arcsin \left(\frac{r}{\rho_{i}(t)}\right) \leq \theta(t) \leq \theta_{i}(t)+\arcsin \left(\frac{r}{\rho_{i}(t)}\right) .
$$

Then, if all constraints over $\theta(t)$ are combined, one obtains

$$
\begin{aligned}
& \max \left(0, \max _{i \in I(t)}\left(\theta_{i}(t)-\arcsin \left(\min \left(1, \frac{r}{\rho_{i}(t)}\right)\right)\right)\right) \leq \theta(t) \\
& \leq \min \left(\frac{\pi}{2}, \min _{i \in I(t)}\left(\theta_{i}(t)+\arcsin \left(\min \left(1, \frac{r}{\rho_{i}(t)}\right)\right)\right)\right) .
\end{aligned}
$$

One could then define the first solution box by

$$
[\boldsymbol{u}]^{(1)}(t)=\left[\underline{\rho}^{(1)}(t), \bar{\rho}^{(1)}(t)\right] \times\left[\underline{\theta}^{(1)}(t), \bar{\theta}^{(1)}(t)\right],
$$




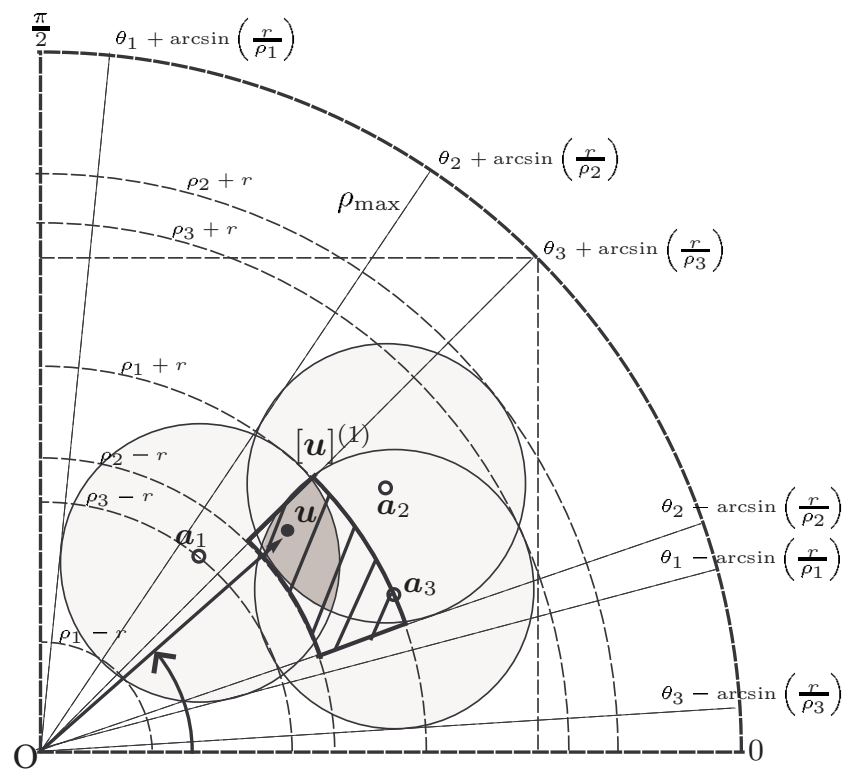

Fig. 4. First solution box obtained for the localization problem of Fig. 2.

with

$$
\begin{aligned}
& \underline{\rho}^{(1)}(t)=\max \left(0, \max _{i \in I(t)}\left(\rho_{i}(t)-r\right)\right) \\
& \bar{\rho}^{(1)}(t)=\min \left(\rho_{\max }, \min _{i \in I(t)}\left(\rho_{i}(t)+r\right)\right) \\
& \underline{\theta}^{(1)}(t)=\max \left(0, \max _{i \in I(t)}\left(\theta_{i}(t)-\arcsin \left(\min \left(1, \frac{r}{\rho_{i}(t)}\right)\right)\right)\right) \\
& \bar{\theta}^{(1)}(t)=\min \left(\frac{\pi}{2}, \min _{i \in I(t)}\left(\theta_{i}(t)+\arcsin \left(\min \left(1, \frac{r}{\rho_{i}(t)}\right)\right)\right)\right)
\end{aligned}
$$

Fig. 4 shows in thick black line the first solution box $[\boldsymbol{u}]^{(1)}(t)$, obtained for the localization problem of Fig. 2. It is obvious that this box is not minimal. It is indeed larger than the solution box that should be obtained, as illustrated in Fig. 3 . For this reason, one could set more constraints on $\rho(t)$ and $\theta(t)$, with bounds being functions of $\theta(t)$ and $\rho(t)$ respectively. Once $[\boldsymbol{u}]^{(1)}(t)$ is computed, these constraints could be used to contract it at maximal leading to the final solution box.

On the first hand, constraints of (6) and equation (11) lead to the following constraints with $i \in I(t)$,

$$
\begin{aligned}
& \rho_{i}(t) \cos \left(\theta(t)-\theta_{i}(t)\right)-\sqrt{r^{2}-\rho_{i}^{2}(t) \sin ^{2}\left(\theta(t)-\theta_{i}(t)\right)} \leq \rho(t) \\
& \leq \rho_{i}(t) \cos \left(\theta(t)-\theta_{i}(t)\right)+\sqrt{r^{2}-\rho_{i}^{2}(t) \sin ^{2}\left(\theta(t)-\theta_{i}(t)\right)} .
\end{aligned}
$$

On the second hand, constraints of (6) could be reformulated as follows,

$$
\cos \left(\theta(t)-\theta_{i}(t)\right) \geq \frac{\rho^{2}(t)+\rho_{i}^{2}(t)-r^{2}}{2 \rho(t) \rho_{i}(t)} .
$$

Then,

$$
\begin{gathered}
\theta_{i}(t)-\arccos \left(\frac{\rho^{2}(t)+\rho_{i}^{2}(t)-r^{2}}{2 \rho(t) \rho_{i}(t)}\right) \leq \theta(t) \\
\leq \theta_{i}(t)+\arccos \left(\frac{\rho^{2}(t)+\rho_{i}^{2}(t)-r^{2}}{2 \rho(t) \rho_{i}(t)}\right)
\end{gathered}
$$

One could use bounds of $\theta(t)$ and (17) to compute new bounds of $\rho(t)$ and bounds of $\rho(t)$ and (19) to compute new bounds of $\theta(t)$, which might contract $[\boldsymbol{u}]^{(1)}(t)$. Practically, in order to contract the polar box $[\boldsymbol{u}]^{(1)}(t)$, the constraints of (17) and (19) are iterated in the interval framework using the forward-backward contractor [22]. This contractor iterates all constraints, while using interval notations, without any prior order until no contraction is possible. The proposed algorithm at a given time $t$ is illustrated in Algorithm 1. Here $\operatorname{Ar}([\boldsymbol{u}](t))$ yields the area of the polar box $[\boldsymbol{u}](t)=$ $[\underline{\rho}(t), \bar{\rho}(t)] \times[\underline{\theta}(t), \bar{\theta}(t)]$, given by

$$
\operatorname{Ar}([\boldsymbol{u}](t))=\frac{(\bar{\theta}(t)-\underline{\theta}(t))\left(\bar{\rho}(t)^{2}-\underline{\rho}(t)^{2}\right)}{2}
$$

The polar box obtained at time $t$ using Algorithm 1 would be at best the box illustrated in Fig. 3, given the problem of Fig. 2.

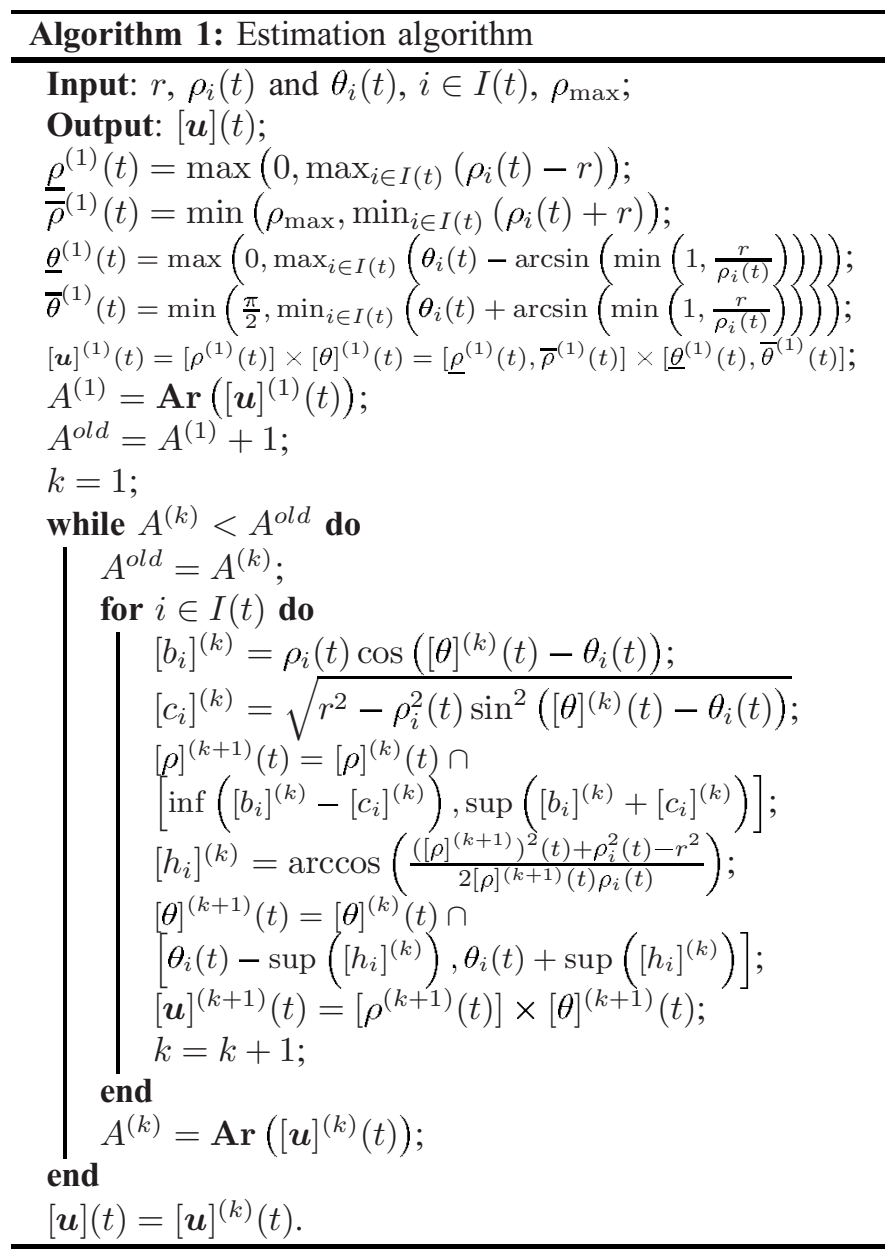

\section{Simulations}

This section illustrates the performances of the proposed method. For this reason, a single mobile node moving over 100 time steps in a $100 \mathrm{~m} \times 100 \mathrm{~m}$ square area is considered. Anchors are assumed to be static and uniformly deployed over the surveillance area. The density of anchors is set to 0.01 anchor per $\mathrm{m}^{2}$, leading to 100 anchors uniformly deployed over the square area, as shown in Fig. 5. With a $10 \mathrm{~m}$ circular sensing range, the node detects at average 3.13 anchors at each time step. Here connectivity measurements at a given time step are generated by computing the distances between the mobile 


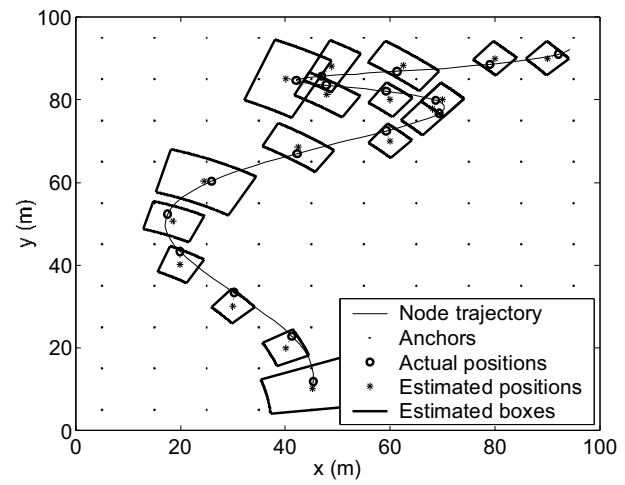

Fig. 5. Estimated boxes obtained using the proposed method.

node and all anchors at this step and then setting to one the measurement corresponding to the anchor having a distance to the node less than $10 \mathrm{~m}$. All simulations are performed on an Intel(R) Atom(TM) CPU (1.67GHz, 1.00GB RAM) using MATLAB 6.1.

\section{A. Illustration of the proposed method}

This paragraph shows an illustration of the proposed method. For this aim, 100 uniformly deployed anchors are considered and the origin of the PCS is assumed to be at the low-left corner of the plot at the cartesian position $(0,0)$. Fig. 5 shows the polar boxes obtained using the proposed method, called PIL. The plot also shows the estimated positions given at the centers of the estimated boxes. The computation time of PIL is equal to $0.0123 s$ at average per time step whereas the average box area is equal to $78.82 \mathrm{~m}^{2}$ per box. Let the estimation error be the average distance between the actual positions of the node and the estimated positions, then the estimation error of PIL is equal to $2.21 \mathrm{~m}$ per time step. A comparison of PIL to its relaxed version, called PILr, is then performed. The PILr method is the simplified version of PIL without the contraction phase, leading to the initial solution box. The average computation time of PILr is equal to $0.00624 \mathrm{~s}$ per time step. The average box area is equal to $79.75 \mathrm{~m}^{2}$ whereas the estimation area is equal to $2.23 \mathrm{~m}$. The use of the contraction phase in PIL leads thus to a slightly higher accuracy, at the cost of the increase of the computation time. The accuracy of both methods varies with the variation of the number and the distribution of anchors in the network. Indeed, with a density of anchors of 0.02 anchor per $\mathrm{m}^{2}$, the average boxes areas obtained with PIL and PILr are equal to $24.61 \mathrm{~m}^{2}$ and $26.78 \mathrm{~m}^{2}$ with computation times of $0.0276 \mathrm{~s}$ and $0.00636 \mathrm{~s}$ respectively. In all cases, PIL yields at least as much accuracy as PILr, with higher computation time. In the following, the relaxed version PILr of the method is considered, since with 100 anchors it leads to almost the same accuracy as PIL with less computation time.

\section{B. Comparison to an interval-based method}

In this section, the proposed method is compared to an interval-based method using cartesian coordinates [21]. This

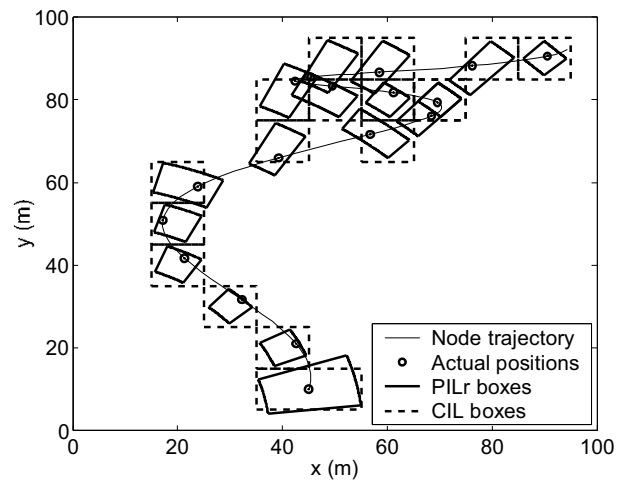

Fig. 6. Estimated boxes obtained using the PILr and the CIL methods.

method, called CIL, yields rectangular boxes estimates covering all possible solutions. Fig. 6 shows the boxes obtained using the relaxed version of the method PILr and the CIL method with a density of anchors of 0.01 anchor per $\mathrm{m}^{2}$. The average computation times are equal to $0.0064 s$ with PILr and $0.0136 s$ with CIL per time step. According to the plot, the boxes of the proposed method are included in the boxes of CIL at most of the time steps. Fig. 7 shows the ratios of the boxes areas obtained with the PILr method over the ones obtained with the CIL method in the top plot and the ratios of the estimation errors obtained with PILr over the ones obtained with CIL in the bottom plot. Note that the estimation error of CIL is equal to the average distance between the actual positions of the node and the centers of the estimated boxes, as for PILr. The average ratio of boxes areas is equal to 0.649 whereas the average ratio of estimation errors is equal to 0.762 . Here the proposed method yields more accurate estimates than the CIL method. The accuracy of both methods is tightly related to the shape of the solution area, which depends on the number and the positions of the anchors in the network. Indeed, assume that the network has 200 anchors randomly deployed over the surveillance area. Here, the average ratios of boxes areas and estimation errors PILr over CIL are equal to 1.045 and 1.096 respectively. One could then propose to perform both techniques for instance and select at each time step the box having the smallest area.

\section{Comparison to a Monte-Carlo-based method}

This section compares the proposed method to a MonteCarlo-based method [14]. This method, called MCL, yields at each time step a fixed number $N$ of positions, called particles, in the way to cover at best the solution area. The estimated positions using MCL correspond to the centers of the computed particles. $N$ is first set to 50 . Fig. 8 shows the estimated particles using MCL as well as the estimated boxes using PILr. With 50 particles, the computation time of MCL is equal to $0.385 \mathrm{~s}$ per time step with an estimation error of $2.762 \mathrm{~m}$. Here the estimation error of MCL is equal to the average distance between the centers of the particles and the actual positions. Compared to PILr, with a computation time of $0.0064 \mathrm{~s}$ and an estimation error of $2.23 \mathrm{~m}$, the MCL is 

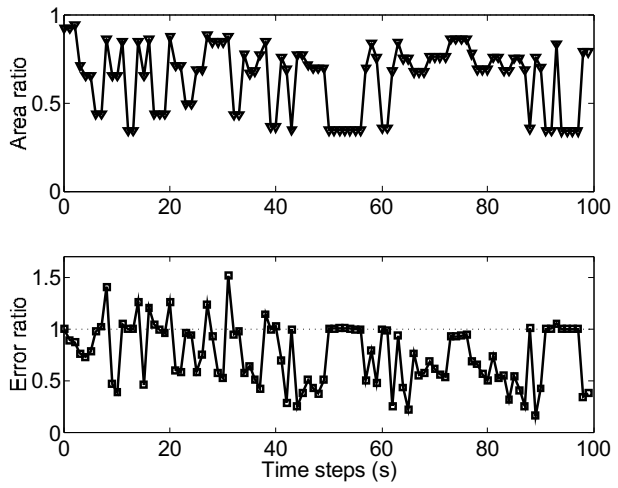

Fig. 7. Ratios of the areas of the boxes obtained with PILr over those of CIL in the top plot. Ratios of the estimation errors obtained with PILr over those of CIL in the bottom plot.

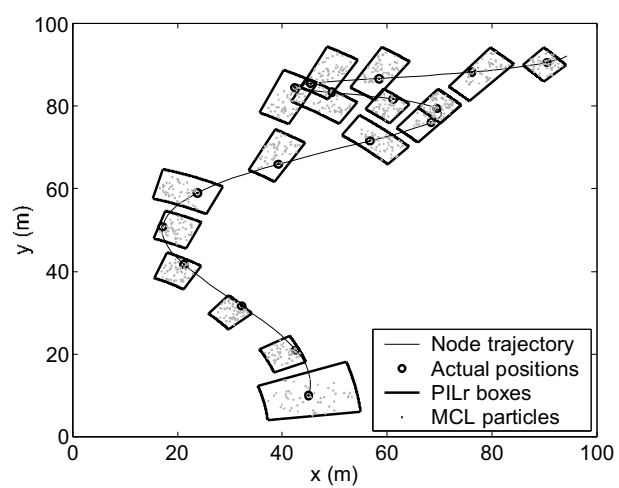

Fig. 8. Estimated boxes with PILr and particles obtained using MCL.

less accurate with more computation time. It is also more consuming in terms of memory resources. The performances of MCL depend on the number of particles to be computed at each time step. Indeed, with $N=20$ for instance, the computation time of MCL decreases to $0.0794 \mathrm{~s}$, at the cost of the estimation error that increases to $3.585 \mathrm{~m}$.

\section{CONCLUSION}

This paper proposes an original technique for sensors localization in wireless sensor networks. The proposed approach is an anchor-based method using connectivity measurements. Based on proximity information, the solution is provided using polar intervals. An outer approximation of the solution is performed using interval analysis in the polar coordinate system. Instead of punctual estimates, the proposed method yields partial rings including for sure all the possible solutions of the problem. Future works will take advantage of the mobility of the nodes to refine the obtained estimates. An extension of this method could also be proposed, with computation performed in different polar coordinate systems.

\section{REFERENCES}

[1] I. F. Akyildiz, W. Su, Y. Sankarasubramaniam, and E. Cayirci, "A survey on sensor networks," IEEE Communications Magazine, vol. 40, pp. $102-$ 114, 2002.

[2] K. Sohraby, D. Minoli, and T. Znati, Wireless sensor networks: technology, protocols, and applications. John Wiley \& Sons, 2007.
[3] R. Shorey, Ed., Mobile, Wireless, and Sensor Networks: Technology, Applications, and Future Directions. John Wiley \& Sons, 2006.

[4] D. Zeinalipour-Yazti and P. K. Chrysanthis, "Mobile sensor network data management," in Encyclopedia of Database Systems, 2009, pp. 17551759.

[5] http://www.mobilerobots.com/ResearchRobots/ResearchRobots.aspx.

[6] F. Mourad, H. Snoussi, F. Abdallah, and C. Richard, "A robust localization algorithm for mobile sensors using belief functions and interval theory," IEEE Transactions on Vehicular Technology, vol. 60, no. 4, pp. 1799-1811, 2011.

[7] M. Ali, A. Böhm, and M. Jonsson, "Wireless sensor networks for surveillance applications - a comparative survey of mac protocols," in The 4th International Conference on Wireless and Mobile Communications, 2008.

[8] A. Czubak and J. Wojtanowski, "On applications of wireless sensor networks," in Internet - Technical Development and Applications, S. B. . Heidelberg, Ed., 2009, pp. 91-99.

[9] B. Hofmann-Wellenhof, H. Lichtenegger, and J. Collins, Global Positioning System: Theory and Practice. Springer, September 2004.

[10] J. Shen and A. Molisch, "Passive location estimation using TOA measurements," in IEEE International Conference on Ultra-Wideband (ICUWB), sept. 2011, pp. 253-257.

[11] R. Eickhoff, F. Ellinger, R. Mosshammer, R. Weigel, A. Ziroff, and M. Huemer, "3D-accuracy improvements for TDoA based wireless local positioning systems," in IEEE GLOBECOM Workshops, nov. 30-dec. 4 2008, pp. $1-6$.

[12] J. Tai, S. Y. Tan, and C. K. Seow, "Three-dimensional non-line-of-sight localisation in an indoor multipath environment," in 7th International Conference on Information, Communications and Signal Processing (ICICS), dec. 2009, pp. 1-5.

[13] J. Tsuji, H. Kawamura, K. Suzuki, T. Ikeda, A. Sashima, and K. Kurumatani, "Zigbee based indoor localization with particle filter estimation," in IEEE International Conference on Systems Man and Cybernetics (SMC), oct. 2010, pp. 1115-1120.

[14] L. Hu and D. Evans, "Localization for mobile sensor networks," in Tenth annual international conference on mobile computing and networking (MobiCom), Philadelphia, USA, 2004.

[15] H. Ren and M.-H. Meng, "Power adaptive localization algorithm for wireless sensor networks using particle filter," IEEE Transactions on Vehicular Technology, vol. 58, no. 5, pp. 2498-2508, jun 2009.

[16] A. Awad, T. Frunzke, and F. Dressler, "Adaptive distance estimation and localization in wsn using rssi measures," in 10th Euromicro Conference on Digital System Design Architectures, Methods and Tools (DSD), aug. 2007, pp. 471-478.

[17] A. Paul and E. Wan, "Rssi-based indoor localization and tracking using sigma-point kalman smoothers," IEEE Journal of Selected Topics in Signal Processing, vol. 3, no. 5, pp. 860-873, oct. 2009.

[18] J. Teng, H. Snoussi, and C. Richard, "Decentralized variational filtering for simultaneous sensor localization and target tracking in binary sensor networks," in IEEE International Conference on Acoustics, Speech and Signal Processing (ICASSP), april 2009, pp. 2233-2236.

[19] M. Mansouri, L. Khoukhi, H. Snoussi, and C. Richard, "Quantized variational filtering for target tracking and relay localization in sensor networks," in 7th International Wireless Communications and Mobile Computing Conference (IWCMC), july 2011, pp. 1837-1842.

[20] F. Mourad, H. Snoussi, F. Abdallah, and C. Richard, "Model-free interval-based localization in manets," in IEEE 13th Digital Signal Processing Workshop and IEEE 5th Signal Processing Education Workshop (DSP/SPE), jan. 2009, pp. 474-479.

[21] - "Anchor-based localization via interval analysis for mobile adhoc sensor networks," IEEE Transactions on Signal Processing, vol. 57, no. 8, pp. 3226-3239, 2009.

[22] L. Jaulin, M. Kieffer, O. Didrit, and E. Walter, Applied interval analysis. Springer, 2001.

[23] A. Medeisis and A. Kajackas, "On the use of the universal okumura-hata propagation prediction model in rural areas," in Vehicular Technology Conference Proceedings, vol. 3, 2000.

[24] Z. Nadir, N. Elfadhil, and F. Touati, "Pathloss determination using okumura-hata model and spline interpolation for missing data for oman," in Proceedings of the World Congress on Engineering, vol. 1, London, UK, July 2008. 